\title{
Apropriação Tecnológica e Design Centrado no Aluno no Contexto de Ambientes Virtuais de Aprendizagem: aprofundando o tema pela perspectiva dos professores
}

\author{
Márcia Coelho Cardoso, Milene Selbach Silveira \\ ${ }^{1}$ PPGCC - PUCRS \\ Av. Ipiranga, 6681, Partenon - Porto Alegre - RS - Brasil
}

\begin{abstract}
The search for improvements in the quality of education leads to interest in technological tools that support the teaching and learning activities in a student-centered design approach. The goal of this study is to explore AVAs activities and resources focusing on how they can improve technological appropriation and student-centered design. The subject is explored starting from studies on the literature, AVAs analysis, and teachers' researches highlighting the relationships between the semiotic affordance levels and technological appropriation ones as well as insights about the gap that exists between what is projected for an AVA and how it is really used.
\end{abstract}

Resumo. A busca por melhorias na qualidade da educação leva a um interesse em ferramentas tecnológicas que apoiem as atividades de ensino e de aprendizagem. $O$ objetivo desse trabalho é explorar atividades e recursos de AVAs compreendendo como eles podem ajudar na apropriação tecnológica e no design centrado no aluno. O tema é explorado a partir de estudos na literatura, análise de AVAs e pesquisas com professores, destacando relações entre os níveis de affordances semióticos e de apropriação tecnológica assim como insights sobre o gap existente entre o que foi projetado para um AVA e a forma como ele é, de fato, utilizado.

\section{Introdução}

Muitas tecnologias são utilizadas nas atividades acadêmicas com o intuito de otimizar o processo de ensino e de aprendizagem tanto para o professor quanto para o aluno. $\mathrm{O}$ professor desempenha um papel importante na maneira como a tecnologia é revelada aos alunos [McCulloch et al. 2018]. Suas escolhas ditam como os alunos são expostos às tecnologias e também têm suas atividades guiadas e acompanhadas. E não basta disponibilizar as tecnologias aos professores na expectativa de integração destas em sala de aula, é preciso que eles saibam como utilizá-las da forma correta e estejam dispostos a lidar com elas [Ottenbreit-Leftwich et al. 2010].

$\mathrm{E}$, em meio aos aspectos envolvidos no processo de aprendizagem com apoio em tecnologias, o design de aprendizagem tem estimulado interesse crescente nos últimos tempos. Além dele, é preciso investigar, ainda, a apropriação tecnológica, que desempenha um papel crucial em relação à aceitação e integração da tecnologia em salas de aula [Jones and Twidale 2005] [Derboven et al. 2017].

Sendo assim, faz-se necessário aprofundar as investigações sobre as escolhas do professor durante o design de aprendizagem, como as ferramentas tecnológicas de apoio 
podem influenciar estas escolhas e melhorar o processo de apropriação tecnológica do professor. Como foco de estudo, foram escolhidos os Ambientes Virtuais de Aprendizagem (AVAs), pelos quais os estudantes podem acessar diretrizes e intervenções pedagógicas com o objetivo de mediar o processo educativo com os colegas e com o professor [Valentini and do Sacramento Soares 2010][Sandtrø 2012][Derboven et al. 2017].

As próximas seções apresentam a fundamentação teórica utilizada como base para este trabalho, estudos iniciais que ajudaram a aprimorar a pesquisa, uma investigação feita com professores para se discutir o tema aqui em questão, uma discussão sobre os resultados encontrados, bem como as considerações finais sobre a pesquisa realizada.

\section{Fundamentação Teórica}

Nem sempre os usuários usam as tecnologias da maneira como estas foram concebidas pelos designers. Várias são as situações em que os usuários adaptam ou alteram tecnologias de acordo com suas necessidades, usando-as para fins não considerados inicialmente [Carroll 2004]. As ações que evidenciam a estruturação desse processo podem ser denominadas de apropriação tecnológica [DeSanctis and Poole 1994].

De acordo com Jones e Twidale (2005), dentre as características de uma aplicação que podem facilitar sua apropriação pelo usuário estão a simplicidade (aplicações simples e diretas facilitam o aprendizado do usuário sobre elas), a ludicidade (a aplicação deve encorajar o usuário a brincar com ela, explorá-la sem uma meta específica), a disponibilidade (física e cognitiva, pois é possível que um aplicativo possua recursos que o usuário desconheça, impossibilitando a apropriação deles), a granularidade (capacidade que a aplicação tem de permitir ao usuário criar composições, e ter seus processo de elaboração reproduzido em momentos distintos para se obter o mesmo fim) e o compartilhamento (permite aos usuários desenvolverem-se entre si, inclusive reutilizando apropriações).

Já Sandtrø (2012) descreve os seguintes níveis como parte desse processo de apropriação:

- Objetificação: quando a aplicação tem um lugar "físico" no computador e na rotina do usuário [Habib and Sønneland 2010];

- Incorporação: quando a utilização da aplicação extrapola um ambiente [Habib and Sønneland 2010];

- Customização: relacionado ao usuário ser capaz de customizar a aplicação de forma a melhor atender seus desejos e necessidades esse aspecto [Habib 2005].

Em referência à apropriação, Derboven, Geerts e De Grooff (2017) enunciam três recomendações sobre o desenvolvimento de AVAs. A primeira propõe que os desenvolvedores devem oferecer um grupo limitado de ferramentas de comunicação aberta, apropriável, com vastas possibilidades de interpretação, além de funcionalidades e ferramentas mais específicas. A recomendação seguinte propõe que o foco deve ser no sentido da comunicação (professor-estudante, estudante-professor ou estudante-estudante). Por fim, a última recomendação propõe que AVAs devem oferecer um ambiente cooperativo de criação, de forma a possibilitar aos professores compartilhar seus conteúdos.

Ainda neste cenário, falhas na comunicação designer-usuário [De Souza 2005] podem levar o usuário a não se apropriar de determinado sistema. E, para que esta 
comunicação se estabeleça, os usuários devem conseguir perceber as affordances da aplicação [De Souza et al. 2000], projetadas pelo designer, as quais podem ser classificadas em três níveis: o nível operacional, relacionado com as ações que o usuário precisa executar; o nível tático, relacionado ao conjunto de ações que o usuário necessita realizar para alcançar determinado objetivo; e, o nível estratégico, relacionado à conceitualização envolvida na formulação do problema e nos processos de resolução desse problema.

O conceito de design de aprendizagem é definido como "a aplicação de um modelo pedagógico para um objetivo de aprendizagem específico, grupo alvo, contexto particular e domínio de conhecimento" [Conole and Fill 2005]. Nesse contexto, existem ambientes de aprendizagem centrados no aluno que são ferramentas tecnológicas moldadas com o objetivo de prover os estudantes com recursos em que eles mesmos possam gerenciar seu aprendizado, através da exploração, formação de hipóteses e troca de feedbacks com outros alunos [Iiyoshi et al. 2005][Land and Hannafin 1996]. No contexto atual, design de aprendizagem, implica também na integração de ferramentas tecnológicas em sala de aula. E, para esta integração existir, é necessário que o uso destas ferramentas seja aceito pelo professor.

Por fim, na última década, vários métodos de ensino emergiram sob a influência da teoria do construtivismo. Essa teoria tem agido como fonte para o desenvolvimento de abordagens focadas no aluno [Baeten et al. 2010], que procuram dar ênfase nas atividades e responsabilidades do aluno ao invés do professor.

\section{Estudos Iniciais}

Para compreender melhor o contexto desta pesquisa, após revisão da literatura sobre apropriação tecnológica e design de aprendizagem, foi aplicado um questionário com o objetivo de aprofundar o entendimento sobre design de aprendizagem, recursos ou atividades tecnológicas utilizadas pelos professores e as barreiras encontradas por eles quando da utilização e integração delas na sua prática diária [Cardoso 2020]. Os resultados para este questionário ressaltaram o uso de AVAs como o Moodle e o GoogleClassroom, bem como o uso (e dificuldades relacionadas a este uso) de recurso como Fóruns, Arquivos e Questionários.

Esses pontos despertaram o interesse em saber mais sobre esses recursos ou atividades, como eles têm contribuído no cenário de design de aprendizagem e de que maneiras é possível aprimorar essa contribuição. Como primeiro passo nesta investigação foi aplicado o Método de Inspeção Semiótica (MIS) [De Souza et al. 2006] sobre o Moodle (versão utilizada na instituição de ensino dos autores) e sobre o GoogleClassroom [Cardoso 2020]. Este método foi escolhido por examinar quão bem a metacomunicação do designer para o usuário chega a este último por meio das interfaces da aplicação sob análise [De Souza et al. 2006], sendo o papel da metacomunicação bastante relevante no processo de apropriação do usuário final, no nosso caso, professores.

Após a aplicação do MIS nas atividades e recursos selecionados dos dois AVAs, alguns pontos se destacaram sobre o alinhamento entre as metamensagens de cada fase, a qualidade da ajuda disponível neles, a sua interface e a complexidade na execução de suas tarefas[Cardoso 2020]. As metamensagens do Moodle foram as menos consistentes e até mesmo contraditórias. Quanto a ajuda, o GoogleClassroom possui uma ajuda mais atualizada e condizente com o comportamento atual da aplicação. Quanto à interface, 
a do GoogleClassroom se mostrou mais simples e eficaz. A simplicidade das janelas do GoogleClassroom tem impacto direto no terceiro item observado: a complexidade na execução das tarefas, que nesse caso foi mais fluída. Já no Moodle, o fato de algumas janelas terem muitas seções de configuração sem informação disponível tornou o processo de execução das tarefas mais demorado e complexo.

Em relação ao design centrado no aluno, os AVAs estudados têm potencial para auxiliar nessa abordagem. Porém, na análise das metamensagens não foram encontradas informações ou pistas que aludissem à mesma. Logo, ficaria a cargo do professor ter o insight de como utilizar a ferramenta nesse sentido.

\section{Aprofundando a investigação com professores}

A fim comparar os resultados até então obtidos com a visão de professores que utilizam os AVAs, a terceira fase da nossa pesquisa foi constituída de entrevistas feitas com esses professores.

Inicialmente foram contatados 19 professores via e-mail, Linkedin ou WhatsApp, em uma amostragem por conveniência. Ao final das tratativas, foi possível realizar, efetivamente, 9 entrevistas. Os detalhes sobre as entrevistas são apresentados a seguir.

\subsection{Perfil dos Participantes}

Quanto aos participantes, conforme mencionado, a amostra foi composta por 9 professores, todos trabalhando em versões institucionais dos AVAs (de colégios de Ensino Médio e Faculdades), com perfis apresentados na Tabela 1.

\begin{tabular}{lllll} 
Código $^{1}$ & Nível $^{2}$ & Uso $^{3}$ & AVA & Área \\
\hline GC1 & Ensino Médio & 2 & GoogleClassroom & Ciências \\
\hline MD2 & Ensino Superior & 9 & Moodle & Letras \\
\hline BB3 & Ensino Superior & 6 & Blackboard & Ciência da Computação \\
\hline BB4 & Ensino Superior & 6 & Blackboard & Ciência da Computação \\
\hline BB5 & Ensino Superior & 7 & Blackboard & Ciência da Computação \\
\hline MD6 & Ensino Superior & 10 & Moodle & Engenharia de Produção \\
\hline MD7 & Ensino Superior & 10 & Moodle & Ciência da Computação \\
\hline MD8 & Ensino Superior & 3 & Moodle & Ciência da Computação \\
\hline GC9 & Ensino Médio & 1 & GoogleClassroom & Informática \\
\hline
\end{tabular}

${ }^{1}$ Para o melhor entendimento sobre a origem dos depoimentos, foi feita a seguinte classificação: BBX participante das entrevistas que usa Blackboard, GCX - participante das entrevistas que usa GoogleClassroom e MDX - participante das entrevistas que usa Moodle. E X representando uma numeração sequencial dos entrevistados, de 1 a 9.

2 Nível que o professor leciona.

${ }^{3}$ Experiência do professor com o AVA (em anos).

Tabela 1. Perfil dos Participantes.

\subsection{Metodologia de coleta e análise de dados}

Dado o contexto da nossa pesquisa e a fim de se atingir os objetivos mencionados previamente, definiu-se a utilização de uma abordagem qualitativa. Como parte dessa aborda- 
gem, foram realizadas entrevistas semiestruturadas ${ }^{1}$, aplicadas de forma individual, sendo elas gravadas em áudio ou vídeo para apoio a posterior análise. O roteiro da entrevista incluía questões sobre apropriação tecnológica, design centrado no aluno, percepções relativas à aceitação da tecnologia em sala de aula e necessidades dos professores em relação aos AVAs.

Para chegar aos significados dos dados de nosso estudo de forma mais efetiva, utilizamos sistemas formais de análise [Rubin and Rubin 2011] em conjunto com nossas questões de pesquisa: codificação para demarcar as ideias nos dados; agrupamento de tipos semelhantes de informação em categorias; e, relações de ideias e temas encontrados.

O passo inicial da análise consistiu na organização dos dados das entrevistas (transcrições dos áudios ou vídeos) de modo a alinhá-los dentro de cada assunto relacionado às perguntas feitas a fim de facilitar a visualização dos dados e a extração de conceitos a partir desses dados.

Em seguida partimos para a codificação desses dados. Esse processo consistiu em assinalar tags ou rótulos a grupos de dados compilados para melhor organizá-los [Miles and Huberman 1994]. Vale ressaltar que esse não foi um processo totalmente sequencial. Ao longo da análise, novos códigos foram emergindo, assim como categorias, que vieram para organizar os grupos de dados dentro dos códigos. Para essa fase, levamos em consideração conceitos pré-estabelecidos durante a pesquisa como as características de design de apropriação tecnológica [Jones and Twidale 2005], os níveis de apropriação tecnológica [Sandtrø 2012], o design centrado no aluno [Hannafin et al. 2009][Derboven et al. 2017] e, também, vimos o surgimento de codificações e categorias não previstas inicialmente, como a codificação "interação" e suas categorias: gamificação, mensagens particulares, vídeos comentados e videoconferência.

Com os dados devidamente organizados, também foi possível definir temas mais abrangentes que pudessem agrupar categorias relacionadas entre si. Finda a parte da codificação dos dados, seguimos para a análise dos resultados cujos detalhes podem ser vistos na seção a seguir.

\subsection{Resultados}

Considerando as características desejadas pelos usuários, a mais citada, apontada por 6 dos 9 participantes, foi a simplicidade (que é também uma das características de apropriação levantadas por Jones e Twidale (2005)). Sobre a simplicidade, BB3 fala em "Facilidade do uso, poder mexer sem ter medo de errar." e BB5 "Mas é muito clica, clica de novo, que abre outra opção, que abre outra opção, que abre outra opção.".

A segunda característica mais desejada foi a de integração com outras ferramentas, apontada por 4 dos 9 participantes, tendo sido citada por entrevistados em cada um dos AVAs pesquisados. BB3 buscava meios de engajar mais seus alunos através do AVA como podemos observar por sua fala "Integração com outras recursos ou atividades com redes sociais...talvez o engajamento dos alunos fosse mais interessante". Demonstrando preocupação com o aspeto administrativo dos AVAs, MD7 cita "Ah, eu acho que

\footnotetext{
${ }^{1}$ Todos os participantes assinaram o Termo de Consentimento Livre e Esclarecido (TCLE) no qual autorizaram o uso dos dados coletados durante as entrevistas para fins acadêmicos. O protocolo de pesquisa ao qual esta pesquisa está associada foi aprovado pelo Comitê de Ética da Universidade dos autores.
} 
IX Congresso Brasileiro de Informática na Educação (CBIE 2020)

Anais do XXXI Simpósio Brasileiro de Informática na Educação (SBIE 2020)

o sistema de atas deveria estar linkado nele. Deveria estar tudo centralizado." e GC9 acrescenta "Eu não consigo integrar as notas deles também com nenhum outro tipo de aplicativo.".

Em seguida, foram detectadas as características de ludicidade e compartilhamento. Em relação à ludicidade, MD6 cita que "Ludicidade, favorece liberdade ao usuário e segurança para realizar exploração." e BB3 fala que um bom AVA proporciona aos usuários "Poder mexer sem ter medo de errar.". Sobre o compartilhamento foram observadas necessidades dos professores em compartilhar conteúdo com outros colegas, a fim aumentar a colaboração e troca de conhecimentos. GC9 nos fala dessa necessidade em "Mas eu não consigo compartilhar a atividade com outros colegas. E isso seria interessante para eles saberem o que eu tô fazendo.".

Em relação à apropriação tecnológica, conseguimos identificar dois grandes grupos. Um com um alto nível de apropriação e outro de nível mediano. O grande diferencial entre os dois foi o quanto o professor incorporou o uso do AVA no seu dia e utilizava recursos ou atividades um pouco mais elaboradas na criação de suas aulas.

No grupo 1, temos alguns depoimentos que demonstram curiosidade e prazer em usar os AVAs. Nos contando sobre suas motivações, MD2 fala "Eu uso ele porque eu gosto de fazer uma aula diferenciada.". No aspecto da curiosidade, BB4 cita "Eu costumo ficar me atualizando e tentando ver novos recursos, possibilidades o tempo todo." e BB5 complementa dizendo "Perto dos outros usuários, eu sou uma usuária avançada. Mas eu sou de futricar.". Já no segundo grupo, temos comportamentos mais discretos. Por exemplo, GC9 demonstra fazer uso do que é estritamente necessário para a execução de suas tarefas quando fala "Não me aventuro muito na aplicação. As coisas que eu precisei, eu tinha prazo e fui atrás. Outra que não precisei, não fui buscar ainda.". Também demonstrando fazer uso de uma porção limitada dos AVAs, BB3 cita "Não sou o maior usuário do AVA. Sempre usei basicamente as mesmas ferramentas.".

Quanto ao design centrado no aluno, a maioria dos participantes respondeu que o AVA disponibilizava meios para se trabalhar com essa abordagem. Para trabalhar dessa forma, BB4 fala "Eu crio um exercício, empacoto SCORM e utilizo. Eu posso fazer sala de aula invertida...". Por sua vez, MD7 conta "Usei o Moodle para disponibilizar o material e já avisava que tinha uma atividade relacionada a esse tema.". Ainda assim, os entrevistados disseram fazer uso de aplicações externas ao AVA para suprir a necessidade por interatividade que o AVA não cobria. BB3 citou usar bastante o Mentimeter enquanto BB5 falou sobre uma experiência integrando o GoogleMaps ao AVA com que trabalha. Destacamos ainda MD7 e MD8 que relataram, respectivamente, "Usei um aplicativo de gamificação que é o Kahoot." e "Eu uso o Kahoot. Porque ele estimula a competição.". Foram relatadas ainda dificuldades externas aos AVAs para o desenvolvimento de atividades com design centrado no aluno, como a falta de tempo por parte do professor, citada por BB3 e GC9, respectivamente, "Os professores têm muitas atividades pra fazer e falta tempo pro professor preparar uma aula mais adequada." e "Tempo que leva pra fazer esse tipo de atividade.".

Foram discutidas, ainda, facilidades e dificuldades disponibilizadas pelos AVAs. No grupo das facilidades, sobre organização de conteúdo e migração de dados entre semestres, BB3 fala que toda a organização de sua disciplina se encontra no AVA e que pode 
migrar os dados de seus cursos de um semestre para outro, apenas fazendo alguns ajustes, sem precisar repensar toda a disciplina a cada semestre. A integração com o diário de classe é um ponto que facilita bastante o trabalho, na visão de BB4. Já BB5 ressalta o papel do AVA em facilitar a distribuição de materiais quando cita "Eu acho que facilita na distribuição de material." sendo acompanhado por MD8 que diz "Era onde eu colocava o material, era onde os alunos olhavam os exercícios, onde os alunos olhavam as datas dos trabalhos, as definições dos trabalhos.". O depoimento de MD8 destaca, também, a comunicação com alunos, que é outra facilidade também mencionada por MD6 " $F a$ cilita para manter contato com os estudantes e também como repositório de atividades desenvolvidos". Por fim, temos o registro de atividades onde BB5 fala "Tu clicou aparece, tu entregou aparece, tu começou mas não terminou, aparece.", citando que isso evita tentativas dos alunos de burlarem os prazos de entrega de atividades e exercícios.

Já no grupo das dificuldades, sobre a organização de conteúdo, GC1 se queixou da interface do GoogleClassroom e que no GoogleDrive "fica tudo misturado.". Já sobre a quantidade de passos necessária para se executar uma tarefa, BB3 fala "Poderia facilitar mais, tem muitos clicks." e é acompanhado por BB4 que diz "O que me incomoda no BB, é muita configuração.”. BB5 se queixou de o AVA ter sido configurado de forma muito engessada, deixando pouco espaço para customização por parte dos professores e GC9 falou sobre a avaliação de notas no GoogleClassroom ser "bem precária.".

Por fim, buscamos investigar de que características ou recursos os professores sentiam falta no AVA. Por seus depoimentos, compreendemos que a maioria dos desejos se relacionam com meios de obter maior interatividade com os alunos através de integração com redes sociais, sistema de videoconferência individual, assim como possibilidades de gamificação.

\section{Considerações finais}

É possível encontrar inúmeras ferramentas tecnológicas com o objetivo de auxiliar professores, instituições de ensino e alunos no processo de ensino e de aprendizagem. Porém, existem vários fatores e personagens com poderes de intensificar ou não a integração dessas ferramentas em sala de aula.

Encontramos relação entre os níveis de affordance operacional e o nível de objetificação de apropriação da seguinte maneira: para se trabalhar com o AVA, o mínimo necessário é que a aplicação exista e esteja disponível ao professor. Por exemplo, temos professores que usam fóruns, videoconferência ou disponibilizam materiais de consulta sobre o assunto estudado como vídeos, links e apresentações através do AVA, rotineiramente. Do ponto de vista do nível de affordance operacional, os usuários conseguem perceber os affordances que os designers dos AVAs disponibilizaram em relação ao uso deles para finalidades específicas, como para disponibilizar vídeos, links, fórum, etc. De forma que os AVAs disponibilizam affordances - no nível de affordance operacional - que afetam os níveis de affordance táticos e estratégicos do que foi planejado pelos professores.

Dentre esses fatores que podem influenciar a incorporação de uma ferramenta, podemos citar a característica de disponibilidade contemplada na lista de Jones e Twidale (2005) sobre apropriação. Se um AVA, por exemplo, é indisponível no acesso remoto, isso pode dificultar ou impedir sua incorporação por parte do usuário. E se De Souza 
et al. (2000) nos fala que o nível de affordance estratégico consiste na conceitualização envolvida nos processos de formulação e de solução para um problema, podemos dizer que uma vez que o usuário não incorporou o uso de uma ferramenta, dificilmente ele vai levá-la em consideração durante essa conceitualização do nível de affordance estratégico. Como exemplo, o usuário pode decidir utilizar outros meios, como o WhatsApp, para notificar os alunos de suas aulas, disponibilizar conteúdo, trocar informações ou tirar dúvidas dos alunos. O WhatsApp está disponível tanto pelo telefone, quanto pela web e ainda possui uma versão desktop. Já os AVAs nem sempre têm esses mesmos recursos habilitados.

Quanto à customização, encontramos relação com os níveis de affordance tático e estratégico. Pois, a não possibilidade de customização de uma ferramenta pode levar o usuário a mudanças no nível de affordance tático que se refletem no nível de affordance estratégico. Nas entrevistas foi dito que um ambiente muito rígido e engessado freia os limites e possibilidades do que o professor pode trabalhar com seus alunos.

Já sobre a percepção de auto-suficiência, encontramos depoimentos de professores que acreditam ter facilidade com os AVAs e crêem que isso se deve ao fato de serem da área de exatas.

Outro aspecto a ser considerado é a qualidade das metamensagens dos AVAs. Em casos em que as metamensagens são inconsistentes, por exemplo, é possível que as funcionalidades desejadas existam, mas não estejam claras ao usuário [Jones and Twidale 2005][De Souza et al. 2000]. Essa tentativa do designer em comunicar uma affordance que não é percebida pelo usuário afeta o nível de incorporação da apropriação, assim como os níveis de affordance operacional e tático, e por consequência o nível de affordance estratégico. De Souza et al. (2000) nos falam que a extensão de possibilidades disponibilizada por um software aos seus usuários não tem muito valor em IHC caso o usuário não perceba essas possibilidades. Essa ideia se relaciona com o conceito de disponibilidade de Jones e Twidale (2005) que descreve que a disponibilidade de uma ferramenta, além de ser física deve ser estar cognitiva também.

Existem ainda situações em que o usuário sabe da existência de uma ferramenta ou recurso na aplicação, mas ache complicado ou confuso demais o seu uso [De Souza et al. 2000]. Podemos citar a Inspeção Semiótica feita nos questionários do Moodle e GoogleClassroom na segunda fase dessa pesquisa. Nela, constatamos discrepâncias entre as diferentes metamensagens, o que pode influenciar percepções como as dos participantes do questionário online e das entrevistas que se queixam de os questionários serem ferramentas muito complexas e por isso preferem evitar o uso deles. Nessas situações temos recursos do AVA que não chegaram ao nível de incorporação, posto que foram deliberadamente recusados pelo professores, e isso afetou direto o nível de affordance estratégico.

Ainda sobre metamensagens, foi citada a necessidade da ferramenta tour. Isso nos faz refletir sobre a influência das ferramentas de ajuda para os níveis de affordance e de apropriação tecnológica. Essa sugestão encontra suporte no trabalho que tem como objetivo a construção de sistemas de ajuda, buscando assegurar que o sistema mostrará aos usuários o que eles podem fazer com a aplicação, a sua finalidade, por que certas decisões de design foram tomadas, dentre outros [Silveira et al. 2004]. Como exemplo, 
no questionário online, houve queixa de que há pouca ajuda e ainda assim é insuficiente para entender como usar a ferramenta de questionários no Moodle.

De modo geral, os professores entendem design centrado no aluno como sendo algo mais interativo e, pelas suas falas, eles acreditam que os AVAs disponibilizam meios para que se trabalhe sob esta perspectiva.

Ficou evidente que o maior gap entre o que foi projetado para um AVA e como ele é, de fato, utilizado se encontra na subutilização que alguns professores fazem deles. Por exemplo, boa parte dos professores afirmam que o maior uso feito dos AVAs é como respositório. Parte desse comportamento pode ser explicado pelas dificuldades encontradas durante a Inspeção Semiótica dos AVAs, que apontou vários desalinhamentos entre as metamensagens que contribuíram para tornar a realização das tarefas mais difíceis.

Um fator bastante citado como barreira à melhor utilização dos AVAs foi o tempo. Esse fator extrapola os limites da tecnologia em si. Mas acreditamos que essa barreira possa ser contornada com o desenvolvimento de sistemas que primem pela simplicidade e ludicidade [Jones and Twidale 2005], tornando a experiência do professor menos árdua. A falta de tempo, associada à percepção de que o AVA não é muito intuitivo ou fácil de usar, como foi observado durante a Inspeção Semiótica e nas entrevistas, contribui para a sua baixa incorporação em sala de aula.

Como sugestão para trabalhos futuros está a pesquisa sob a perspectiva do aluno, investigando a visão destes sobre os temas aqui discutidos.

\section{Agradecimentos}

O presente artigo foi alcançado em cooperação com a Hewlett Packard Brasil LTDA., com a HP Brasil Indústria e Comércio de Equipamentos Eletrônicos LTDA. e com recursos provenientes da Lei de Informática (Lei $n^{\circ} 8.248$, de 1991). Também houve apoio da Uol EdTech.

\section{Referências}

Baeten, M., Kyndt, E., Struyven, K., e Dochy, F. (2010). Using student-centred learning environments to stimulate deep approaches to learning: Factors encouraging or discouraging their effectiveness. Educational Research Review, 5(3):243-260.

Cardoso, M. (2020). Apropriação tecnológica e design centrado no aluno no contexto de ambientes virtuais de aprendizagem. Dissertação de mestrado, Programa de PósGraduação em Ciência da Computação, PUCRS, Porto Alegre, RS, Brasil.

Carroll, J. (2004). Completing design in use: closing the appropriation cycle. Em European Conference of Information Systems, page 44.

Conole, G. e Fill, K. (2005). A learning design toolkit to create pedagogically effective learning activities. Journal of Interactive Media in Education, 2005(1):1-16.

De Souza, C. S. (2005). The semiotic engineering of human-computer interaction. MIT press.

De Souza, C. S., Leitão, C. F., Prates, R. O., e Da Silva, E. J. (2006). The semiotic inspection method. Em Symposium on Human Factors in Computing Systems, pages 148-157. ACM. 
IX Congresso Brasileiro de Informática na Educação (CBIE 2020)

Anais do XXXI Simpósio Brasileiro de Informática na Educação (SBIE 2020)

De Souza, C. S., Prates, R. O., e Carey, T. (2000). Missing and declining affordances: Are these appropriate concepts? Journal of the Brazilian Computer Society, 7(1):26-34.

Derboven, J., Geerts, D., e De Grooff, D. (2017). Appropriating virtual learning environments: A study of teacher tactics. Journal of Visual Languages and Computing, 40:20-35.

DeSanctis, G. e Poole, M. S. (1994). Capturing the complexity in advanced technology use: Adaptive structuration theory. Organization Science, 5(2):121-147.

Habib, L. (2005). Finding a place and a space for online learning environments in an institutional setting: issues of objectification. Transactions on Internet Research, 1(2):713 .

Habib, L. e Sønneland, A. M. (2010). From alien to domestic? Virtual learning environment use from a domestication perspective. Journal of Online Learning and Teaching, 6(4):712-722.

Hannafin, M., Hannafin, K., e Gabbitas, B. (2009). Re-examining cognition during student-centered, Web-based learning. Educational Technology Research and Development, 57(6):767-785.

Iiyoshi, T., Hannafin, M. J., e Wang, F. (2005). Cognitive tools and student-centred learning: rethinking tools, functions and applications. Educational Media International, 42(4):281-296.

Jones, M. C. e Twidale, M. B. (2005). What's in a name? Exploring the connections between abstraction and appropriation. International reports on socio-informatics, 2(2):43-47.

Land, S. M. e Hannafin, M. J. (1996). Student-Centered Learning Environments: Foundations, Assumptions, and Implications, chapter 1, pages 3-25. Taylor \& Francis.

McCulloch, A. W., Hollebrands, K., Lee, H., Harrison, T., e Mutlu, A. (2018). Factors that influence secondary mathematics teachers' integration of technology in mathematics lessons. Computers \& Education, 123:26-40.

Miles, M. B. e Huberman, A. M. (1994). Qualitative data analysis: An expanded sourcebook. Sage Publications.

Ottenbreit-Leftwich, A. T., Glazewski, K. D., Newby, T. J., e Ertmer, P. A. (2010). Teacher value beliefs associated with using technology: Addressing professional and student needs. Computers \& Education, 55(3):1321-1335.

Rubin, H. J. e Rubin, I. S. (2011). Qualitative interviewing: The art of hearing data. Sage Publications.

Sandtrø, T. A. (2012). How the domestication process of a VLE came to closure. The Online Educational Research Journal, 3:2-8.

Silveira, M. S., Barbosa, S. D. J., e Souza, C. S. d. (2004). Designing online help systems for reflective users. Journal of the Brazilian Computer Society, 9:25 - 38.

Valentini, C. B. e do Sacramento Soares, E. M. (2010). Aprendizagem em ambientes virtuais: compartilhando idéias e construindo cenários. EDUCS. 